\title{
Analytical study for time and time-space fractional Burgers' equation
}

\author{
$\mathrm{KM} \mathrm{Saad}^{1,2^{*}}$ and Eman HF Al-Sharif ${ }^{1,2}$
}

\author{
"Correspondence: \\ Khaledma_sd@hotmail.com \\ 'Department of Mathematics, \\ College of Arts and Sciences, Najran \\ University, Najran, 61441, Saudi \\ Arabia \\ 2 Department of Mathematics, \\ Faculty of Applied Science, Taiz \\ University, Taiz, Yemen
}

\begin{abstract}
In this paper, the variational iteration method (VIM) is applied to solve the time and space-time fractional Burgers' equation for various initial conditions. VIM solutions are computed for the fractional Burgers' equation to show the behavior of VIM solutions as the fractional derivative parameter is changed. The results obtained by VIM are compared with exact solutions and also with expansions of the exact solutions. VIM solutions are found to be in excellent agreement with these exact solutions.
\end{abstract}

Keywords: variational iteration method; space-time fractional Burgers equation; different initial values

\section{Introduction}

In recent years fractional calculus has been utilized to find solutions of equations governing the modeling of real materials in engineering and physics. Fractional differential equations model many phenomena in several fields such as fluid mechanics, chemistry [1, 2], biology [3], viscoelasticity [4], engineering, finance, and physics [5-7]. Therefore, the use of fractional calculus has gained the attention of scientists and engineers as, though counter-intuitive, the fractional derivative does arise in physical problems. In this connection there is the work of [8-15]. Due to the difficulty of obtaining exact solutions of equations involving a fractional derivative, approximate and numerical techniques have tended to be used instead. Examples of such approximate and numerical methods are Taylor collocation method followed in [16]; Adomian's decomposition method followed in $[17,18]$; finite difference method followed in $[19,20]$; homotopy analysis method and homotopy perturbation methods followed, respectively, in [21-23] and [24].

In addition, there are general papers giving an overview of the field of fractional differential equations [25-28]. Also, numerical methods have been used to find solutions of fractional differential equations [29-37].

Burgers' equation is the diffusive equation

$$
u_{t}+a u u_{x}-c u_{x x}=0 .
$$

Burgers' equation arises as a model equation for the smoothing of a shock wave by viscosity [38]. In this application $c$ is modeled on the kinematic viscosity in the Navier-Stokes equations. Furthermore, Burgers' equation has an exact solution via the Cole-Hopf equation which reduces it to the heat equation $[37,38]$. Of particular relevance to this work,

(c) The Author(s) 2017. This article is distributed under the terms of the Creative Commons Attribution 4.0 International License (http://creativecommons.org/licenses/by/4.0/), which permits unrestricted use, distribution, and reproduction in any medium, provided you give appropriate credit to the original author(s) and the source, provide a link to the Creative Commons license, and indicate if changes were made. 
Burgers' equation (1) has the front solution [39-41]

$$
u(x, t)=\frac{2 b}{a}-\frac{2 c b}{a} \tanh [b(x-2 b t)]
$$

In this paper we study approximate solutions using VIM for the time and space-time fractional Burgers' equation. To the best of our knowledge, this is the first study of the space-time fractional Burgers' equation by VIM. The work of $[42,43]$ studied only the time fractional Burgers' equation and the space fractional Burgers' equation, but did not study the space-time fractional Burgers' equation.

The present paper is organized as follows. The second and third sections are devoted to the basic ideas of fractional calculus and the standard VIM, respectively. The fourth and fifth sections are devoted to the application of VIM to evaluate solutions of the time and space-time fractional Burgers' equation, respectively. Conclusions are presented in section seven.

\section{Fractional calculus}

Definitions of fractional derivatives were given by Riemann, Liouville, Grunwald, Letnikov and Caputo [25-28], and they are based on generalized functions. The most commonly used definitions are those of Riemann [25] and Liouville and Caputo [27]. Here we give some basic definitions and properties of fractional calculus theory.

Definition 2.1 A real function $f(t), t>0$, is said to be in the space $C_{\mu}, \mu \in R$, if there exists a real number $p>\mu$ such that $f(t)=t^{p} f_{1}(t)$, where $f_{1}(t) \in C[0, \infty)$, and it is said to be in the space $C_{\mu}^{m}$ iff $f^{m} \in C_{m}, m \in N$.

The Riemann-Liouville fractional integral operator of order $\alpha>0$ for a function $f \in C_{\mu}$, $\mu \geq-1$, is defined as

$$
\begin{aligned}
& J_{0}^{\alpha} f(t)=\frac{1}{\Gamma(\alpha)} \int_{0}^{t}(t-\tau)^{\alpha-1} f(\tau) d \tau, \quad t>0, \\
& J^{0} f(t)=f(t) .
\end{aligned}
$$

It has the following properties. For $f \in C_{\mu}, \mu \geq-1, \alpha, \beta \geq 0$, and $\gamma>-1$,

- $J^{\alpha} J^{\beta} f(t)=J^{\alpha+\beta} f(t)$

- $J^{\alpha} J^{\beta} f(t)=J^{\beta} J^{\alpha} f(t)$

- $J^{\alpha} t^{\gamma}=\frac{\Gamma(\gamma+1)}{\Gamma(\alpha+\gamma+1)} t^{\alpha+\gamma}$.

Definition 2.2 The fractional derivative of $f(t)$ in the Caputo sense is defined as

$$
D^{\alpha} f(t)=J^{m-\alpha} D^{m} f(t)=\frac{1}{\Gamma(m-\alpha)} \int_{0}^{t}(t-\tau)^{m-\alpha-1} f^{(m)}(\tau) d \tau
$$

for $m-1<\alpha \leq m, m \in N, t>0, f \in C_{\mu}^{m}, \mu \geq-1$. Then

$$
\begin{aligned}
& D^{\alpha} J^{\alpha} f(t)=f(t), \\
& J^{\alpha} D^{\alpha} f(t)=f(t)-\sum_{k=0}^{m-1} f^{k}\left(0^{+}\right) \frac{t^{k}}{k !} .
\end{aligned}
$$




\section{Basic ideas of VIM}

The principles of VIM and its applications to various kinds of differential equations are given in [44-49]. To apply VIM, we consider the differential equation

$$
L u(x, t)+N u(x, t)=g(x, t)
$$

where $L$ is a linear operator, $N$ is a nonlinear operator and $g(x, t)$ is a source term. According to VIM, we construct the correction functional as

$$
u_{n+1}(x, t)=u_{n}(x, t)+\int_{0}^{t} \lambda\left(L u_{n}(x, \tau)+N \tilde{u}_{n}(x, \tau)-g(x, \tau)\right) d \tau,
$$

where $\lambda$ is a general Lagrange multiplier [45-47] which can be determined optimally through variational theory. The subscript $n$ denotes the $n$th order approximation, whereas $\tilde{u}_{n}(x, t)$ is considered as a restricted variation [45-47], i.e., $\delta \tilde{u}_{n}(x, t)=0$. Also new results were obtained in $[50,51]$ using VIM.

\section{The time-fractional Burgers' equation}

In this section, we use VIM to solve the time-fractional Burgers' equation. To obtain this equation, we replace $u_{t}$ by $u_{t}^{\alpha}$ in Burgers' equation (1), where $n-1<\alpha \leq n, n \in N$. Hence, we obtain the fractional-time Burgers' equation

$$
u_{t}^{\alpha}+a u u_{x}-c u_{x x}=0
$$

Now, we construct the correction functional to solve (6) according to VIM as follows:

$$
\begin{aligned}
& u_{n+1}(x, t)=u_{n}(x, t)+J_{t}^{\alpha} \lambda(\tau)\left(u_{n, \tau}^{\alpha}+a \tilde{u}_{n} \tilde{u}_{n, x}-c u_{n, x x}\right), \\
& u_{n+1}(x, t)=u_{n}(x, t)+\frac{1}{\Gamma(\alpha)} \int_{0}^{t}(t-\tau)^{(\alpha-1)} \lambda(\tau)\left(u_{n, \tau}^{\alpha}+a \tilde{u}_{n} \tilde{u}_{n, x}-c u_{n, x x}\right) d \tau,
\end{aligned}
$$

where $\tilde{u}_{n}, \tilde{u}_{n, x}$ and $\tilde{u}_{n, x x}$ are considered as restricted variations. The correction functional (8) can be approximately expressed as

$$
u_{n+1}(x, t)=u_{n}(x, t)+\int_{0}^{t} \lambda(\tau)\left(u_{n, \tau}^{m}+a \tilde{u}_{n} \tilde{u}_{n, x}-c \tilde{u}_{n, x x}\right) d \tau \text {. }
$$

For $m=1$, we obtain the stationary condition

$$
\begin{aligned}
& \delta u_{n+1}(x, t)=\left.\delta u_{n}(x, t)(1+\lambda(\tau))\right|_{t=\tau}+\int_{0}^{t} \delta u_{n}(x, \tau) \lambda^{\prime}(\tau) d \tau=0, \\
& \lambda^{\prime}(\tau)=0, \\
& \left.(1+\lambda(\tau))\right|_{\tau=t}=0 .
\end{aligned}
$$

Equation (11) is the Euler-Lagrange equation and (12) is the corresponding natural boundary condition. Thus we have $\lambda=-1$, and we obtain the variational iteration formula

$$
u_{n+1}(x, t)=u_{n}(x, t)-\frac{1}{\Gamma(\alpha)} \int_{0}^{t}(t-\tau)^{(\alpha-1)}\left(u_{n, \tau}^{\alpha}+a u_{n} u_{n, x}-c u_{n, x x}\right) d \tau .
$$


Now, as $n \rightarrow \infty$, we consider $u_{n}$ as an approximation of the exact solution. Iteration (13) is started with $u_{0}(x, t)=u(x, 0)$, and we obtain the successive approximations

$$
\begin{aligned}
u_{0}(x, t)= & \frac{2(b-b c \tanh (b x))}{a}, \\
u_{1}(x, t)= & \frac{2 b}{a \alpha \Gamma(\alpha)}\left(\Gamma(\alpha)(\alpha-\alpha c \tanh (b x))+2 b^{2} c t^{\alpha} \operatorname{sech}^{2}(b x)\right), \\
u_{2}(x, t)= & \frac{2 b}{a \Gamma(\alpha)}\left(\Gamma(\alpha)+\frac{2 b^{2} c t^{\alpha} \operatorname{sech}^{2}(b x)}{\alpha}+8 b^{4} c t^{2 \alpha} \tanh (b x) \operatorname{sech}^{2}(b x)\right. \\
& \left.\times\left(\frac{\sqrt{\pi} 4^{-\alpha}}{\alpha \Gamma\left(\alpha+\frac{1}{2}\right)}+\frac{4 b^{2} c \Gamma(2 \alpha) t^{\alpha} \operatorname{sech}^{2}(b x)}{\Gamma(\alpha+1) \Gamma(3 \alpha+1)}\right)-c \Gamma(\alpha) \tanh (b x)\right) .
\end{aligned}
$$

In this manner, we can obtain further approximations $u_{3}(x, t), u_{4}(x, t), \ldots$ For brevity, these are not given. Note that if we expand the exact solution (2) in a Taylor series about $t=0$, we thus obtain VIM solutions at $\alpha=1$. In Figure 1, we show the surface of the exact solution (2) and the second approximation (16) for $\alpha=0.7$ in (b) at $a=1, b=0.01$ and $c=1$. We note from this figure that the trajectory of the second approximation approaches the exact solution. In Figure 2 we show the absolute error between the exact solution (2) and the second approximation (16) for different values of $b$. The value of $b$ plays a very important role in the acceleration of convergence. As $b$ deceases, the absolute error becomes very small.

\section{The space-time fractional Burgers' equation}

\subsection{Tanh initial condition}

In this section we study VIM solutions of the space-time fractional Burgers' equation. However, before this we expand the exact solution (2) in the basis functions $e^{-r b(x-2 b t)}$. By definition

$$
\begin{aligned}
u(x, t) & =\frac{2 b}{a}-\frac{2 c b}{a} \tanh [b(x-2 b t)]=\frac{2 b}{a}-\frac{2 c b}{a} \frac{1-e^{-2(b(x-2 b t))}}{1+e^{-2(b(x-2 b t))}} \\
& =\frac{2 b}{a}-\frac{2 c b}{a}\left(1+2 \sum_{r=1}^{\infty}(-1)^{r} e^{-2 r(b(x-2 b t))}\right) \\
& =h-\frac{4 c b}{a}\left(\sum_{r=1}^{\infty}(-1)^{r} e^{-2 r(b(x-2 b t))}\right), \quad b(x-2 b t)>0,
\end{aligned}
$$

where $h=\frac{2 b}{a}-\frac{2 c b}{a}$. For convenience, we rewrite Burgers' equation after substituting $u=$ $v+h$ as

$$
v_{t}+a v v_{x}+a h v_{x}-c v_{x x}=0 .
$$

The solution of this equation (18) is

$$
v(x, t)=\frac{-4 c b}{a}\left(\sum_{r=1}^{\infty}(-1)^{r} e^{-2 r(b(x-2 b t))}\right) .
$$


(a)

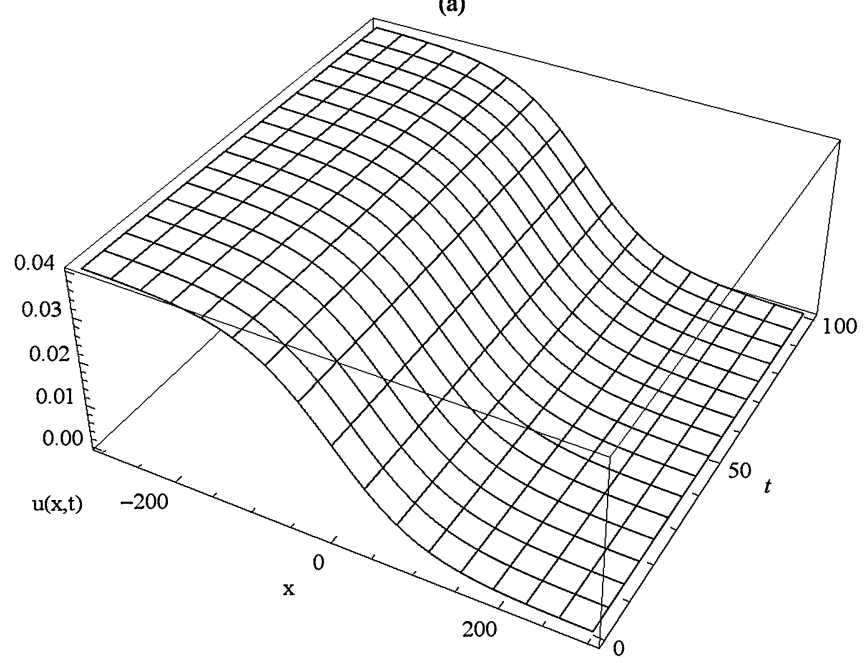

(b)

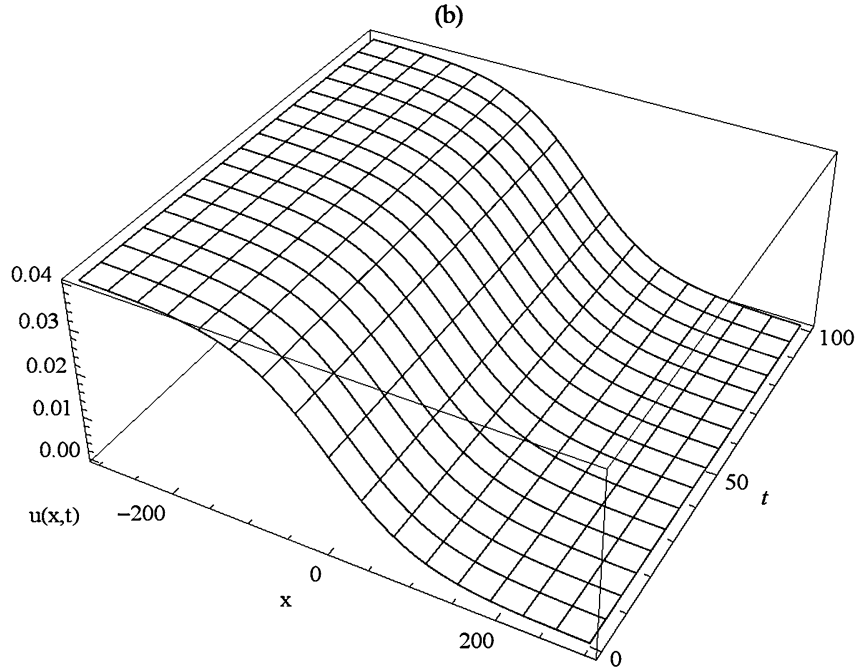

(c)

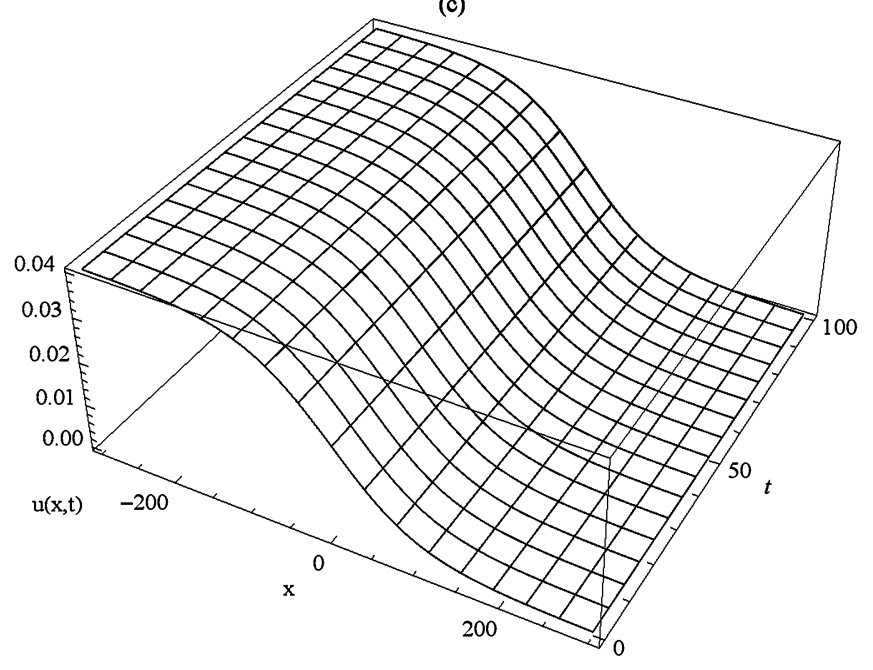

Figure 1 Combining the surface exact solution (2) and the surface approximation (16) for $\alpha=0.7$,

$a=1, b=0.01$, and $c=1$. (a) The surface of the exact solution. (b) The surface of the second approximation. (c) Combining (a)-(b). 


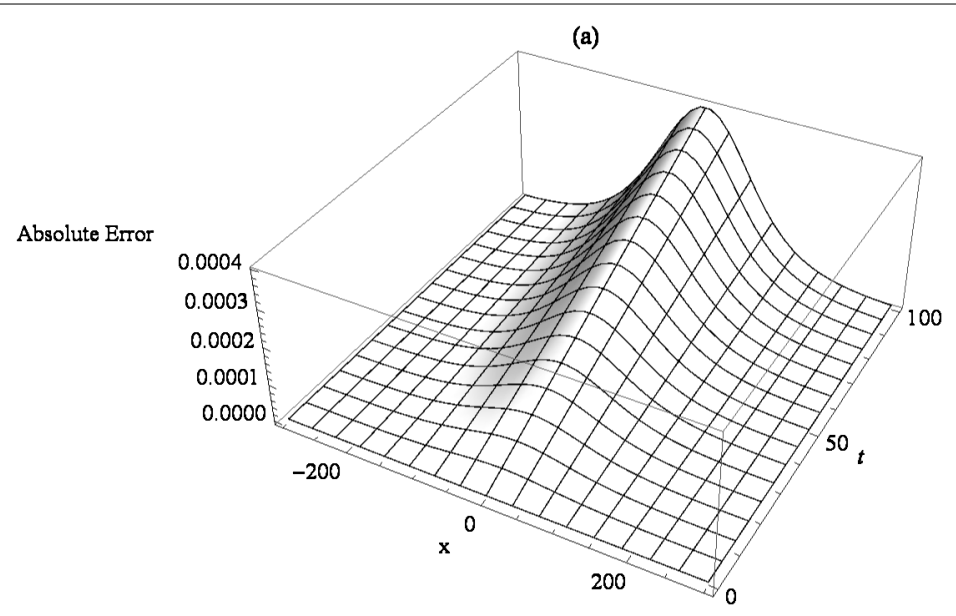

(b)

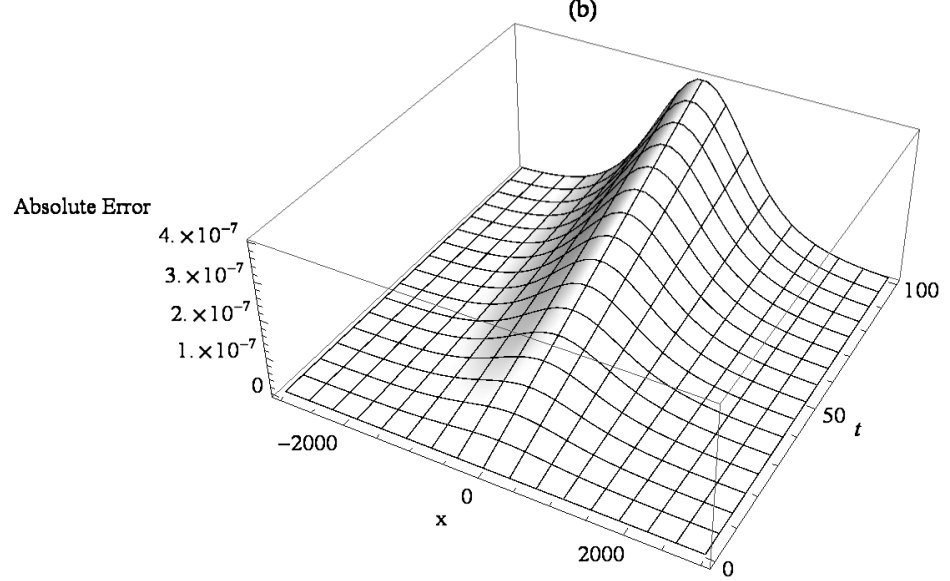

Figure 2 Comparison of the second approximation (16) with the exact solution (2) for $\alpha=1, a=1$, $c=1$ with (a) $b=0.01$ and (b) $b=0.001$.

We take the initial condition from (19) on setting $t=0$

$$
v(x, 0)=\frac{-4 c b}{a}\left(\sum_{r=1}^{\infty}(-1)^{r} e^{-2 r(b x)}\right) .
$$

We shall now study VIM for the space-time fractional Burgers' equation. If we replace $v_{t}$ by $v_{t}^{\alpha}, v_{x}$ by $v_{x}^{\beta}$ and $v_{x x}$ by $v_{x}^{2 \beta}$, where $0<\alpha, \beta \leq 1$, in the time fractional Burgers' equation (18), we obtain

$$
v_{t}^{\alpha}+a(v+h) v_{x}^{\beta}-c v_{x}^{2 \beta}=0
$$

VIM is given by

$$
v_{n+1}(x, t)=v_{n}(x, t)-\frac{1}{\Gamma(\alpha)} \int_{0}^{t}(t-\tau)^{(\alpha-1)}\left(v_{n, \tau}^{\alpha}+a\left(v_{n}+h\right) v_{n, x}^{\beta}-c v_{n, x}^{2 \beta}\right) d \tau .
$$


On using this, we can evaluate the successive approximations. We choose $v_{0}(x, t)=v(x, 0)$ and find

$$
v_{0}(x, t)=\frac{-4 c b}{a}\left(\sum_{r=1}^{\infty}(-1)^{r} e^{-2 r(b x)}\right)
$$

and

$$
v_{1}(x, t)=v_{0}(x)-\frac{t^{\alpha}}{\Gamma(1+\alpha)} f_{0}(x)
$$

The second approximation is given by

$$
\begin{aligned}
& \begin{aligned}
v_{2}(x, t) & =v_{1}(x, t)-\left(\frac{-t^{\alpha} f_{0}}{\Gamma(1+\alpha)}+a I_{1}-c I_{2}\right), \\
I_{1}= & \frac{\left(h+v_{0}\right) g_{1}}{\Gamma(1+\alpha)} t^{\alpha}-\frac{\left(f_{1} g_{1}+f_{2} h \Gamma(\alpha+1)+f_{2} v_{0}(x)\right)}{\Gamma(2 \alpha+1)} t^{2 \alpha} \\
& +\frac{f_{1} f_{2} \Gamma(2 \alpha+1)}{\Gamma(\alpha+1)^{2} \Gamma(3 \alpha+1)} t^{3 \alpha}, \\
I_{2}= & \frac{g_{2}}{\Gamma(\alpha+1)} t^{\alpha}-\frac{\Gamma(\alpha+1) f_{3}}{\Gamma(2 \alpha+1)} t^{2 \alpha}, \\
f_{i}(x)= & \frac{(-4 b c)^{2}}{a} \sum_{r 1=1}^{\infty} \sum_{r=1}^{\infty} \lambda^{\beta}(-1)^{r+r 1}(\lambda+\lambda 1)^{i \beta} e^{x(\lambda+\lambda 1)} \\
& -4 h b c \sum_{r=1}^{\infty}(-1)^{r} \lambda^{(i+1) \beta} e^{\lambda x}+\frac{4 b c^{2}}{a} \sum_{r=1}^{\infty}(-1)^{r} \lambda^{(i+2) \beta} e^{\lambda x}, \quad i=0,1,2,3, \\
g_{i}=- & \frac{4 b c}{a} \sum_{r=1}^{\infty}(-1)^{r} \lambda^{i \beta} e^{\lambda x}, \quad i=1,2, \lambda=-2 b r, \lambda_{1}=-2 b r_{1} .
\end{aligned}
\end{aligned}
$$

In the same manner, we can obtain further approximations $v_{3}(x, t), \ldots$ Setting $\beta=1$ in the first approximation (24) and the second approximation (25) and after some simplifications, we obtain the same approximations as (15) and (16) on returning to the original variable. We can obtain VIM solutions of (21) for $b(x-2 b t)<0$ using the same procedure as for $b(x-2 b t)>0$. Using Mathematica, we can simplify the first approximation $v_{1}$ as

$$
\begin{aligned}
v_{1}(x, t)= & \frac{4 b c}{a\left(e^{2 b x}+1\right)}-\left(\frac{4 b c t^{\alpha}}{a \alpha \Gamma(\alpha)\left(e^{2 b x}+1\right)^{3}}\left(-4 b^{2} e^{2 b x}\left((c+1) e^{2 b x}+3 c+1\right)\right)\right. \\
& \left.-\left(4^{\beta} c(-b)^{2 \beta}\left(e^{2 b x}+1\right)^{3} \text { PolyLog }\left[-2 \beta,-e^{-2 b x}\right]\left(-e^{-2 b x}\right)\right)\right) .
\end{aligned}
$$

We now use the first approximation (30), after returning to the original variable, to find VIM solutions. Figure 3 shows VIM solution (30) for different values of $\alpha$ and $\beta$. It can be seen from this figure that as $\alpha \rightarrow 1$ and $\beta \rightarrow 1$, VIM solution (30) tends to the exact solution (2). Therefore, VIM is an efficient and accurate method which can be used to find approximate analytical solutions of the space-time fractional Burgers' equation. 


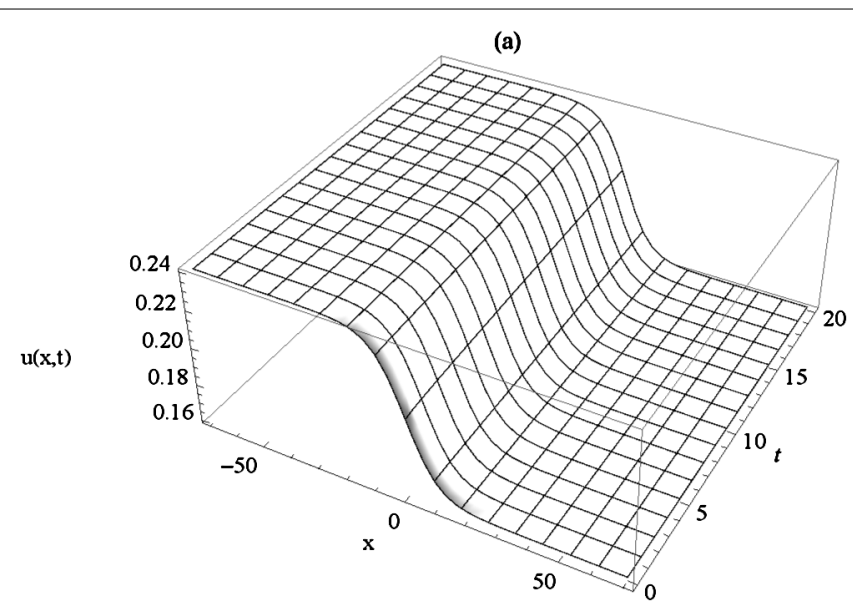

(b)

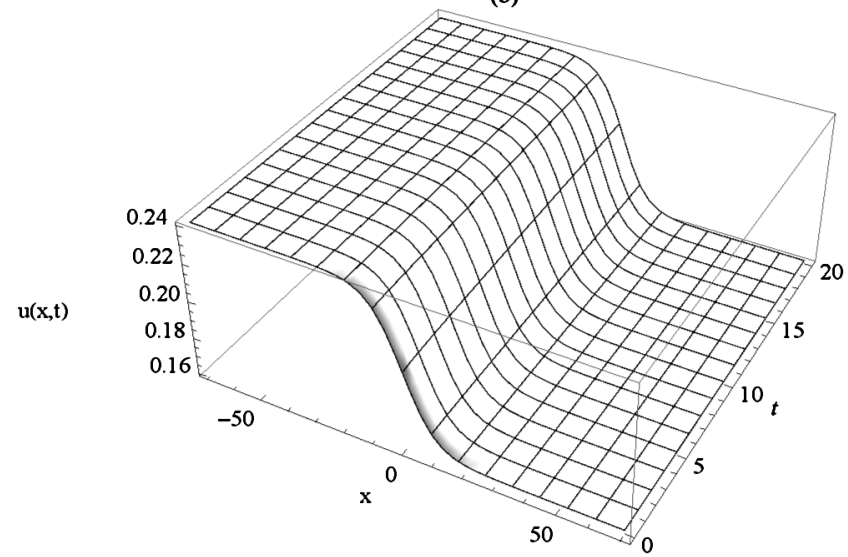

(c)

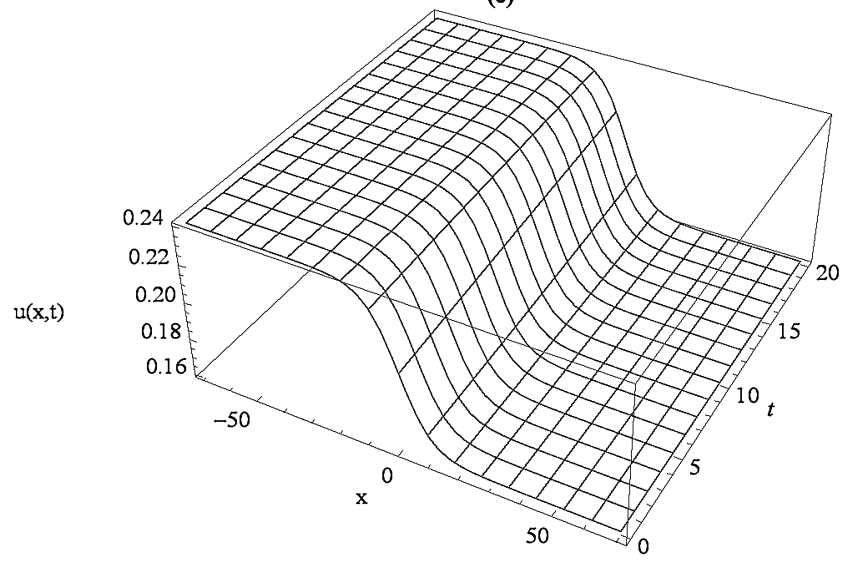

Figure 3 VIM solution (30) in the origin variable $u$ for $a=1, b=0.01$ and $c=0.7$ (a) $\alpha=0.4, \beta=0.6$, (b) $\alpha=0.6, \beta=0.8$, (c) $\alpha=1, \beta=1$ and (d) combining (a)-(c).

\subsection{Polynomial initial condition}

In this section we study VIM for the space-time fractional Burgers' equation for another initial condition. We replace $u_{t}$ by $u_{t}^{\alpha}, u_{x x}$ by $u_{x}^{2 \beta}$ and $u_{x}$ by $u_{x}^{\beta}$, where $0<\alpha, \beta \leq 1$ in Burgers' equation (1). VIM for (1) is

$$
u_{n+1}(x, t)=u_{n}(x, t)-\frac{1}{\Gamma(\alpha)} \int_{0}^{t}(t-\tau)^{(\alpha-1)}\left(u_{n, \tau}^{\alpha}+a u_{n} u_{n, x}^{\beta}-c u_{n, x}^{2 \beta}\right) d \tau .
$$




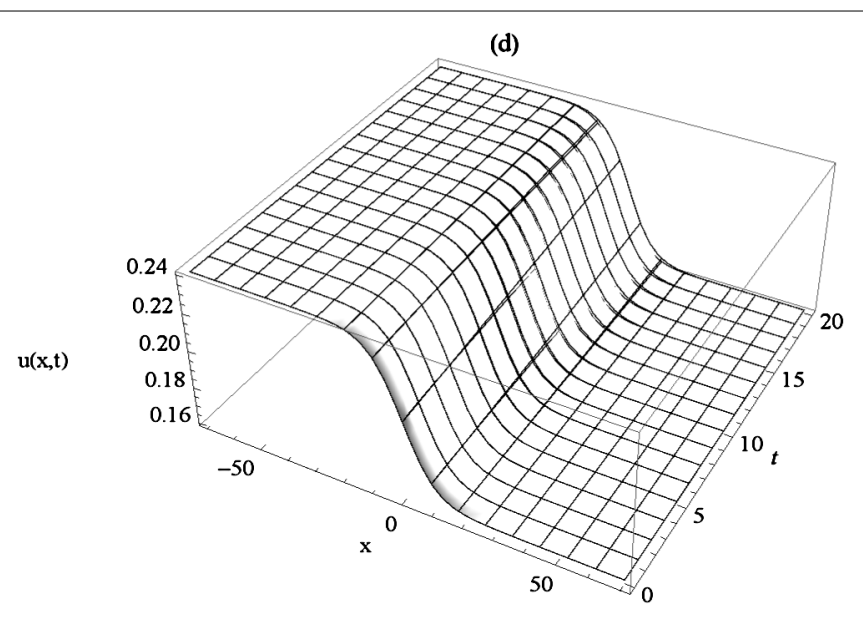

Figure 3 Continued

We start the iteration with $u_{0}(x, t)=u(x, 0)=2 x$, so that the successive approximations can be derived from (31) as

$$
\begin{aligned}
& u_{0}(x, t)=2 x, \\
& u_{1}(x, t)=\left(2 x+\frac{2 t x^{1-2 \beta}}{\Gamma(\alpha)}\left(-\frac{2 a x^{\beta+1}}{\Gamma(2-\beta)}+\frac{c}{\Gamma(2-2 \beta)}\right)\right), \\
& u_{2}(x, t)=u_{1}(x, t)+\frac{1}{\Gamma(\alpha)}\left(b_{1}-\frac{1}{3} 2 a t\left(b_{2}+b_{3}\right) x^{2-3 \beta}+b_{4}\right) \text {, } \\
& b_{1}=\frac{-4 a c t^{2} x^{2-3 \beta}}{\Gamma(\alpha) \Gamma(3-3 \beta)}+\frac{2 a \beta c t^{2} x^{2-3 \beta}}{\Gamma(\alpha) \Gamma(3-3 \beta)}+\frac{c^{2} t^{2} x^{1-4 \beta}}{\Gamma(\alpha) \Gamma(2-4 \beta)}+\frac{2 c t x^{1-2 \beta}}{\Gamma(2-2 \beta)}, \\
& b_{2}=\frac{3 t\left(-\frac{2 a x^{\beta+1}-\frac{c \Gamma(2-\beta)}{\Gamma(2-2 \beta)}}{\Gamma(2-\beta)^{2}}+\frac{2 a(\beta-2) x^{\beta+1}}{\Gamma(3-2 \beta)}+\frac{c}{\Gamma(2-3 \beta)}\right)}{\Gamma(\alpha)}+\frac{6 x^{2 \beta}}{\Gamma(2-\beta)}, \\
& b_{3}=\frac{2 t^{2}}{\Gamma(\alpha)^{2}}\left(\frac{-c x^{-2 \beta}\left(\frac{2 a x^{\beta+1}}{\Gamma(2-\beta)}-\frac{c}{\Gamma(2-2 \beta)}\right)}{\Gamma(2-3 \beta)}+2 a\right. \\
& \left.\times\left(\frac{\frac{a x}{(\beta-1)^{2} \Gamma(-\beta)}+\frac{(\beta-2) c x^{-\beta}}{\Gamma(3-2 \beta)}}{\Gamma(2-2 \beta)}+\frac{4 a x}{\Gamma(3-2 \beta) \Gamma(2-\beta)}\right)\right), \\
& b_{4}=\frac{2 \sin (\pi \alpha) t^{2-\alpha} x^{1-2 \beta}\left(2 a \Gamma(2-2 \beta) x^{\beta+1}-c \Gamma(2-\beta)\right)}{\pi(\alpha-2)(\alpha-1) \Gamma(2-2 \beta) \Gamma(2-\beta)}, \\
& u_{3}(x, t)=u_{2}(x, t)+\cdots,
\end{aligned}
$$

In a similar manner, we calculate further approximations for (31). When we set $\alpha=1$ and $\beta=1, a=1, b=1$ and $c=1$ in (33)-(39), we obtain

$$
\begin{aligned}
& u_{1}(x, t)=2 x(1-2 t) \\
& u_{2}(x, t)=2 x\left(1-2 t+4 t^{2}-\frac{8 t^{3}}{3}\right) \\
& u_{3}(x, t)=2 x\left(1-2 t+4 t^{2}-8 t^{3}+\frac{32}{3} t^{4}-\frac{32}{3} t^{5}+\frac{64}{9} t^{6}-\frac{128}{63} t^{7}\right) \cdots
\end{aligned}
$$


Using the fact that

$$
u(x, t)=\lim _{n \rightarrow \infty} u_{n}(x, t)
$$

we obtain an exact solution of Burgers' equation (1). This solution is the same as that of [42].

Figure 4 shows the surface of the exact solution (43) in (a) and the surface of the second approximation (34) for $\alpha=0.7$ and $\beta=0.5$ in (b) at $a=1, b=1$ and $c=1$. It can be seen from this figure that the second approximate solution is a good approximation of the exact solution. If we go to higher order approximations, we will obtain better approximations to this exact solution.

\section{Convergence analysis}

In this section the existence of a unique solution is introduced in Theorem 6.2. Furthermore, the convergence of VIM solution (22) is proved in Theorem 6.3. Finally, the maximum absolute truncation error of VIM solution (22) is given in Theorem 6.4. In this section we prove theorems for the space-time fractional Burgers' equation. This theorem covers the time-fractional Burgers' equation on setting $\beta=1$. We define $(C(I),\|\cdot\|)$ as a Banach space, the space of all continuous functions on $I=R \times R^{+}$with the norm $\|v(x, t)\|=\max _{(x, t) \in I}|v(x, t)|$.

Lemma 6.1 Suppose that $v(x, t)$ and their partial derivatives are continuous. Then the derivatives $D_{t}^{\alpha} v(x, t), D_{x}^{\beta} v(x, t)$ and $D_{x}^{2 \beta} v(x, t)$ are bounded.

Proof We prove that $D_{t}^{\alpha} v(x, t)$ is bounded. From the fractional derivative definition (3) we have

$$
\begin{aligned}
\left\|D_{t}^{\alpha} v(x, t)\right\| & =\left\|\frac{1}{\Gamma(m-\alpha)} \int_{a}^{b}(t-\tau)^{m-\alpha-1} v^{(m)}(x, \tau) d \tau\right\| \\
& \leq \frac{|b-a|}{|(m-\alpha) \Gamma(m-\alpha)|}\|v(x, t)\|=L_{1}\|v(x, t)\|,
\end{aligned}
$$

where $L_{1}=\frac{|b-a|}{|(m-\alpha) \Gamma(m-\alpha)|}$. In the same manner $\left\|D_{x}^{\beta} v(x, t)\right\| \leq L_{2}\|v(x, t)\|$ and $\left\|D_{x}^{2 \beta} v(x, t)\right\| \leq$ $L_{3}\|v(x, t)\|$.

Theorem 6.2 Let $F(v)=-a v v_{x}^{\beta}$ satisfy the Lipschitz condition with the Lipschitz constant $L_{4}$. Then problem (21) has the unique solution $v(x, t)$ whenever $0<\gamma<1$.

Proof Let $u$ and $v$ be two different solutions of the space-time fractional Burgers' equation (21). For all $t \in[0, T]$ and $\tau \in[0, t]$, these solutions are bounded on using Lemma 6.1. Let $M=\max _{0 \leq \tau \leq t, 0 \leq t \leq T}\left|(t-\tau)^{\alpha-1}\right|$. Then

$$
\begin{aligned}
u-v= & J_{t}^{\alpha}\left(-a h D_{x}^{\beta} u(x, \tau)+c D_{x}^{2 \beta} u(x, \tau)+F(u)\right) \\
& -J_{t}^{\alpha}\left(-a h D_{x}^{\beta} v(x, \tau)+c D_{x}^{2 \beta} v(x, \tau)+F(v)\right) \\
= & \frac{1}{\Gamma(\alpha)} \int_{0}^{t}(t-\tau)^{\alpha-1}\left[-a h D_{x}^{\beta} u(x, \tau)+c D_{x}^{2 \beta} u(x, \tau)+F(u)\right] d \tau \\
& -\frac{1}{\Gamma(\alpha)} \int_{0}^{t}(t-\tau)^{\alpha-1}\left[-a h D_{x}^{\beta} v(x, \tau)+c D_{x}^{2 \beta} v(x, \tau)+F(v)\right] d \tau,
\end{aligned}
$$


(a)

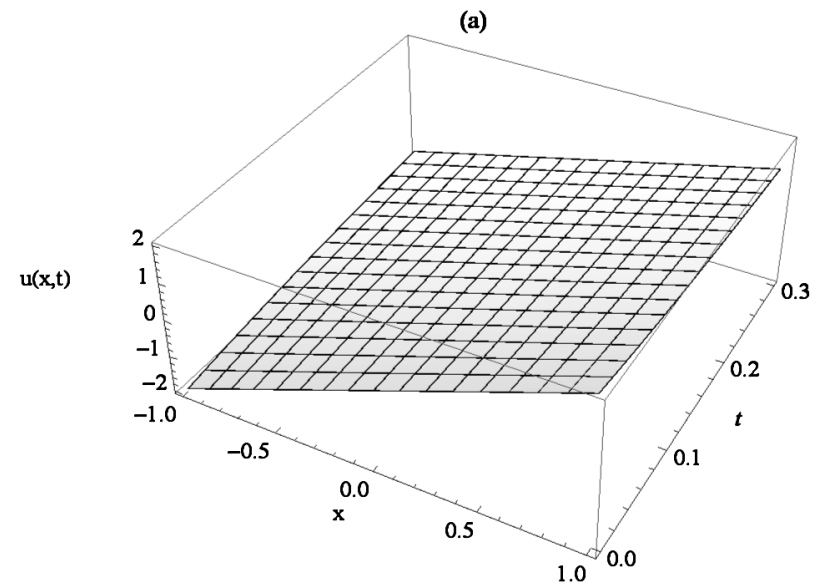

(b)

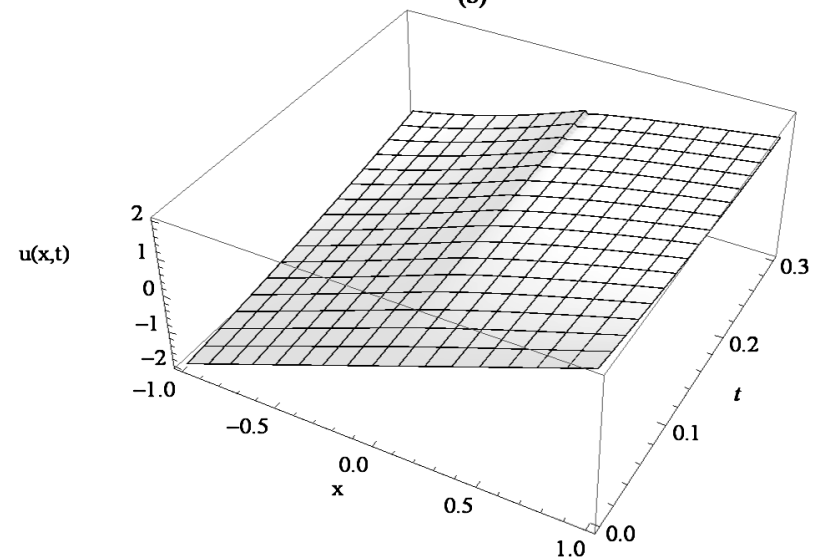

(c)

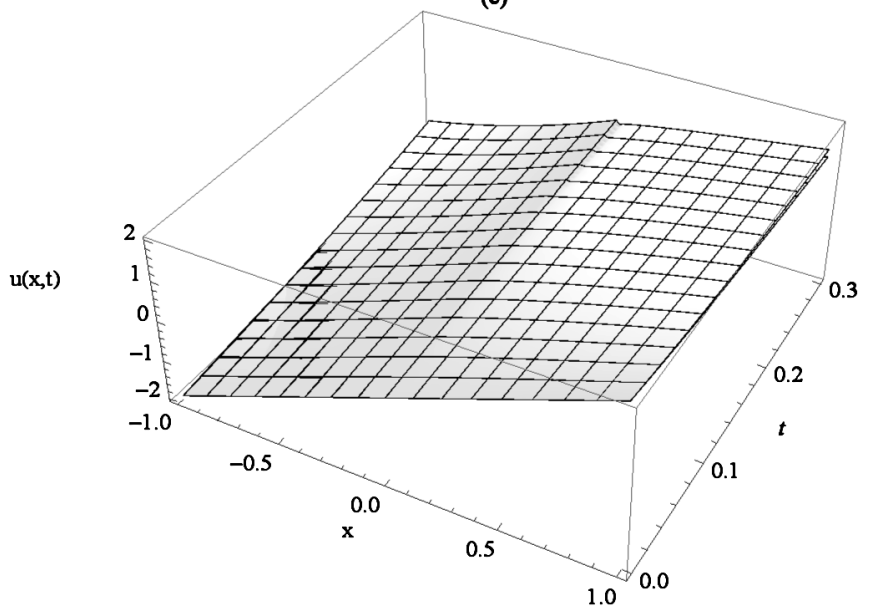

Figure 4 Combining the surface exact solution (43) and the surface approximation (34) for $\alpha=0.7$, $\beta=0.5, a=1, b=1$, and $\boldsymbol{c}=\mathbf{1}$. (a) The surface of the exact solution. (b) The surface of the second approximation. (c) Combining (a)-(b). 


$$
\begin{aligned}
& \max |u-v|= \max \mid \frac{1}{\Gamma(\alpha)} \int_{0}^{t}(t-\tau)^{\alpha-1}\left[-a h D_{x}^{\beta} u(x, \tau)+c D_{x}^{2 \beta} u(x, \tau)+F(u)\right] d \tau \\
& \quad-\frac{1}{\Gamma(\alpha)} \int_{0}^{t}(t-\tau)^{\alpha-1}\left[-a h D_{x}^{\beta} v(x, \tau)+c D_{x}^{2 \beta} v(x, \tau)+F(v)\right] d \tau \mid \\
& \leq \frac{\left[-a h L_{2}+c L_{3}+L_{4}\right]}{\Gamma(\alpha)} \max \int_{0}^{t}|t-\tau|^{\alpha-1}|u-v| d \tau, \\
&\|u-v\| \leq \frac{\left[-a h L_{2}+c L_{3}+L_{4}\right]}{\Gamma(\alpha)} \max \int_{0}^{t}|t-\tau|^{\alpha-1}|u-v| d \tau, \\
&\|u-v\| \leq \gamma\|u-v\|, \\
&(1-\gamma)\|u-v\| \leq 0,
\end{aligned}
$$

where $\gamma=\left(\frac{\left[-a h L_{2}+c L_{3}+L_{4}\right] M T}{\Gamma(\alpha)}\right)$. Since $1-\gamma \neq 0,\|u-v\|=0$. Therefore, $u=v$, which completes the proof.

Theorem 6.3 The sequence $v_{n}(x, t)$ obtained from VIM iteration (22) converges to the exact solution of problem (21) for $0<\sigma<1$ and $0<\gamma_{1}<1$.

Proof The approximate solution using VIM is given by

$$
v_{n+1}(x, t)=v_{n}(x, t)-\frac{1}{\Gamma(\alpha)} \int_{0}^{t}(t-\tau)^{(\alpha-1)}\left(D_{\tau}^{\alpha} v_{n}+a h D_{x}^{\beta} v_{n}-c D_{x}^{2 \beta} v_{n}-F\left(v_{n}\right)\right) d \tau .
$$

Since $v$ is the exact solution of equation (21), it satisfies VIM formula, i.e.,

$$
v(x, t)=v(x, t)-\frac{1}{\Gamma(\alpha)} \int_{0}^{t}(t-\tau)^{(\alpha-1)}\left(D_{\tau}^{\alpha} \nu+a h D_{x}^{\beta} \nu-c D_{x}^{2 \beta} v-F(v)\right) d \tau .
$$

On subtracting $v$ from $v_{n+1}$ and recalling that $E_{n}(x, t)=v_{n}(x, t)-v(x, t)$, we obtain

$$
\begin{aligned}
& E_{n+1}(x, t)=E_{n}(x, t)-\frac{1}{\Gamma(\alpha)} \int_{0}^{t}(t-\tau)^{\alpha-1} \\
& \quad \times\left[D_{t}^{\alpha}\left(v_{n}-v\right)+a h D_{x}^{\beta}\left(v_{n}-v\right)-c D_{x}^{2 \beta}\left(v_{n}-v\right)-F\left(v_{n}-v\right)\right] d \tau \\
& \max \left|E_{n+1}(x, t)\right| \leq \max \left|E_{n}(x, t)\right|-\frac{\left[L_{1}+a h L_{2}-c L_{3}-L_{4}\right] M}{\Gamma(\alpha)} \max \int_{0}^{t}\left|E_{n}(x, \tau)\right| d \tau, \\
& \left\|E_{n+1}(x, t)\right\| \leq\left\|E_{n}(x, t)\right\|-\frac{\left[L_{1}+a h L_{2}-c L_{3}-L_{4}\right] M T}{\Gamma(\alpha)}\left\|E_{n}(x, t)\right\| .
\end{aligned}
$$

Hence,

$$
\left\|E_{n+1}(x, t)\right\| \leq \sigma\left\|E_{n}(x, t)\right\|
$$

where $\sigma=\left(1-\gamma_{1}\right), \gamma_{1}=\frac{\left[L_{1}+a h L_{2}-c L_{3}-L_{4}\right] M T}{\Gamma(\alpha)}$ for all $n=0,1,2, \ldots$ Now, if $n=0$,

$$
\left\|E_{1}(x, t)\right\| \leq \sigma\left\|E_{0}(x, t)\right\|
$$

while if $n=1$,

$$
\left\|E_{2}(x, t)\right\| \leq \sigma^{2}\left\|E_{0}(x, t)\right\| .
$$


If $n=2$, then

$$
\begin{aligned}
& \left\|E_{3}(x, t)\right\| \leq \sigma^{3}\left\|E_{0}(x, t)\right\|, \ldots \\
& \left\|E_{n}(x, t)\right\| \leq \sigma^{n}\left\|E_{0}(x, t)\right\| .
\end{aligned}
$$

Since $0<\sigma<1$, then $\left\|E_{n}(x, t)\right\| \rightarrow 0$ as $n \rightarrow \infty$, i.e., $v_{n} \rightarrow v$ and the sequence $\left\{v_{n}(x, t)\right\}_{n=1}^{\infty}$ converges to $v(x, t)$.

Theorem 6.4 The maximum absolute truncation error of the approximate solution $v_{n}(x, t)$ of the time-space fractional Burgers' equation (21) can be estimated as $\left\|E_{n}(x, t)\right\| \leq$ $\frac{\sigma^{n}}{1-\sigma}\left\|v_{1}(x, t)\right\|$.

Proof

$$
\begin{aligned}
v_{n}(x, t)-v_{n+1}(x, t) & =\left(v_{n}(x, t)-v(x, t)\right)+\left(v(x, t)-v_{n+1}(x, t)\right) \\
& =E_{n}(x, t)-E_{n+1}(x, t) .
\end{aligned}
$$

Hence,

$$
\begin{aligned}
E_{n}(x, t)= & E_{n+1}(x, t)-\left(v_{n+1}(x, t)-v_{n}(x, t)\right), \\
\left\|E_{n}(x, t)\right\| & =\left\|E_{n+1}(x, t)-\left(v_{n+1}(x, t)-v_{n}(x, t)\right)\right\| \\
\left\|E_{n}(x, t)\right\| & \leq\left\|E_{n+1}(x, t)\right\|+\left\|\left(v_{n+1}(x, t)-v_{n}(x, t)\right)\right\| \\
& \leq \sigma\left\|E_{n}(x, t)\right\|+\left\|\left(v_{n+1}(x, t)-v_{n}(x, t)\right)\right\| .
\end{aligned}
$$

Therefore,

$$
\begin{aligned}
\left\|E_{n}(x, t)\right\| & \leq \frac{\left\|\left(v_{n+1}(x, t)-v_{n}(x, t)\right)\right\|}{1-\sigma} \\
& \leq \frac{\sigma^{n}}{1-\sigma}\left\|v_{1}(x, t)\right\| .
\end{aligned}
$$

\section{Conclusions}

Approximate solutions of the time and space-time fractional Burgers' equation have been evaluated using the VIM for different initial conditions by expanding the tanh initial condition in the basis functions $e^{-n x}$. The fractional derivative could then be easily calculated. An important point is that many authors avoid this initial condition as there was no direct method to calculate its fractional derivative. In Figure 1, the surface of VIM solutions with the exact solution of Burgers' equation for $\alpha=0.7$ was plotted. Also, the absolute error with $\alpha=1$ for different values for $b$ were displayed in Figure 2. The effect of changes of $b$ was clear through the resulting decrease of the error. As the value of $b$ decreases, the absolute error becomes very small. In Figure 3 the VIM solutions were plotted with different values of $\alpha$ and $\beta$. The VIM solutions approach the exact solution as the values of $\alpha$ and $\beta$ approach 1, as shown in Figure 4. The results were shown to be in very good agreement with both exact solutions and previous approximate solutions. The calculations in this paper were performed using Mathematica 9. 


\section{Acknowledgements}

We thank Noel Frederick Smyth, Ji-Huan He and Saeid Abbasbandy for stimulating discussions during the preparation of this article.

\section{Competing interests}

The authors declare that they have no competing interests.

\section{Authors' contributions}

All authors drafted the manuscript, and they read and approved the final version of the manuscript.

\section{Publisher's Note}

Springer Nature remains neutral with regard to jurisdictional claims in published maps and institutional affiliations.

\section{Received: 13 June 2017 Accepted: 11 September 2017 Published online: 26 September 2017}

\section{References}

1. Kirchner, JW, Neal, C, Feng, X: Fractal stream chemistry and its implications for contaminant transport in catchments. Nature 403, 524-527 (2000)

2. Giona, M, Roman, HE: Fractional diffusion equation for transport phenomena in random media. Phys. A, Stat. Mech. Appl. 185, 87-97 (1992)

3. Magin, RL: Fractional Calculus in Bioengineering. Begell House, Conn, USA, Danbury (2006)

4. Podlubny, I: Fractional Differential Equations, Mathematics in Science and Engineering. Academic Press, San Diego (1999)

5. Hilfer, R: Applications of Fractional Calculus in Physics. Word Scientific, Singapore (2000)

6. Li, C, Deng, W: Remarks on fractional derivatives. Appl. Math. Comput. 187, 777-784 (2007)

7. Popolizio, M, Garrappa, R: On the use of matrix functions for fractional partial differential equations. Math. Comput. Simul. 81(5), 1045-1056 (2011)

8. Bhrawy, AH: A new spectral algorithm for time-space fractional partial differential equations with subdiffusion and superdiffusion. Proc. Rom. Acad., Ser. A 17, 39-47 (2016)

9. Ali, AM, Inc, EK, Dumitru, B: Numerical solutions of fractional differential equations of Lane-Emden type by an accurate technique. Adv. Differ. Equ. 69, 220 (2015)

10. Inc, M: Exact and numerical solitons with compact support for nonlinear dispersive $k(m, p)$ equations by the VIM. Physica A 375, 447-456 (2007)

11. Inc, M: Numerical doubly periodic solution of the (2+1)-dimensional Boussinesq equation with initial conditions by the VIM. Phys. Lett. A 366, 20-24 (2007)

12. Inc, M: Numerical simulation of kdv and mkdv equations with initial conditions by the VIM. Chaos Solitons Fractals 34 , 1071-1081 (2007)

13. Inc, M, Bulent, $\mathrm{K}$ : The first integral method for the time fractional Kaup-Boussinesq system with time dependent coefficient. Appl. Math. Comput. 254, 70-74 (2015)

14. Inc, M, Bulent, K, Yavuz, U, Dumitru, B: Improved ( $\left.g^{\prime} / g\right)$-expansion method for the time-fractional biological population model and Cahn-Hilliard equation. J. Comput. Nonlinear Dyn. 10, 70-74 (2015)

15. Abdelkawy, MA, Zaky, MA, Bhrawy, AH, Baleanu, D: Numerical simulation of time variable fractional order mobile-immobile advection-dispersion model. Rom. Rep. Phys. 67, 773-791 (2015)

16. Yöcel, Ç, Yildiray, K, Aydin, K: The solution of the Bagley Torvik equation with the generalized Taylor collocation method. J. Franklin Inst. 347(2), 452-466 (2010)

17. Khodabakhshi, N, Vaezpour, SM, Baleanu, D: Numerical solutions of the initial value problem for fractional differential equations by modification of the Adomian decomposition method. Fract. Calc. Appl. Anal. 20(1), 382-400 (2014)

18. Shi, XC, Huang, LL, Zeng, Y: Fast Adomian decomposition method for the Cauchy problem of the time-fractional reaction diffusion equation. Adv. Mech. Eng. 8(2), 1-5 (2016)

19. Fu, H, Wang, $\mathrm{H}$ : A preconditioned fast finite difference method for space-time fractional partial differential equations. Fract. Calc. Appl. Anal. 20(1), 88-116 (2017)

20. Takeuchi, Y, Yoshimoto, Y, Suda, R: Second order accuracy finite difference methods for space-fractional partial differential equations. J. Comput. Appl. Math. 320, 101-119 (2017)

21. Liao, SJ: On the homotopy analysis method for nonlinear problems. Appl. Math. Comput. 147, 499-513 (2004)

22. Saad, KM, AL-Shomrani, AA: An application of homotopy analysis transform method for Riccati differential equation of fractional order. J. Fract. Calc. Appl. 7(1), 61-72 (2016)

23. Saad, KM, AL-Shareef, EH, Mohamed, SM, Yang, XJ: Optimal q-homotopy analysis method for time-space fractional gas dynamics equation. Eur. Phys. J. Plus 132(1), 23 (2017)

24. Liao, SJ: Comparison between the homotopy analysis method and homotopy perturbation method. Appl. Math. Comput. 169, 1186-1194 (2005)

25. Podlubny, I: Fractional Differential Equations. Academic Press, San Diego (1999)

26. Miller, KS, Ross, B: An Introduction to the Fractional Calculus and Fractional Differential Equations. Wiley, New York (1993)

27. Caputo, M: Linear models of dissipation whose q is almost frequency independent. Part II. Geophys. J. Int. 13, 529-539 (1976)

28. Trujillo, JJ, Kilbas, AA, Srivastava, HM: Theory and Applications of Fractional Differential Equations. North-Holland Mathematical Studies, vol. 204. Elsevier, Amsterdam (2006)

29. Agila, A, Baleanu, D, Eid, R, Irfanoglu, B: Applications of the extended fractional Euler-Lagrange equations model to freely oscillating dynamical systems. Rom. J. Phys. 61, 350-359 (2016)

30. Aslan, EC, Inc, M: Numerical solutions and comparisons for nonlinear time fractional Ito coupled system. J. Comput. Theor. Nanosci. 13, 1-6 (2016) 
31. Kumar, D, Singh, J, Baleanu, D: A fractional model of convective radial fins with temperature-dependent thermal conductivity. Rom. Rep. Phys. 69, 103 (2017)

32. Inc, M, Bulent, K: Classification of travelling wave solutions fort the time-fractional fifth-order KdV-like equation. Waves Random Complex Media 24, 393-403 (2014)

33. Nouri, K, Elahi-Mehr, S, Torkzadeh, L: Investigation of the behavior of the fractional Bagley-Torvik and Basset equations via numerical inverse Laplace transform. Rom. Rep. Phys. 68, 503-514 (2016)

34. Fairouz, T, Inc, M, Zeliha, SK, Dumitru, B: Solutions of the time fractional reaction-diffusion equations with residual power series method. Adv. Mech. Eng. 8, 1-10 (2016)

35. Abd-Elhameed, WM, Youssri, YH: Spectral solutions for fractional differential equations via a novel Lucas operational matrix of fractional derivatives. Rom. J. Phys. 61, 795-813 (2016)

36. Yang, XJ, Gao, F, Srivastava, HM: New rheological models within local fractional derivative. Rom. Rep. Phys. 69, 113 (2017)

37. Zhang, Y, Baleanu, D, Yang, XJ: New solutions of the transport equations in porous media within local fractional derivative. Proc. Rom. Acad., Ser. A 17, 230-236 (2016)

38. Whitham, GB: Linear and Nonlinear Waves. Wiley, New York (1974)

39. Feng, Z: Travelling wave solutions and proper solutions to the two-dimensional Burgers-Korteweg-de Vries equation. J. Phys. A, Math. Gen. 36, 8817-8827 (2003)

40. Feng, Z: On travelling wave solutions of the Burgers-Korteweg-de Vries equation. Nonlinearity 20, 343-356 (2007)

41. Feng, Z, Zheng, S, Angew, Z: Traveling wave solutions to a reaction-diffusion equation. Math. Phys. 60, 756-773 (2009)

42. Biazar, J, Aminikh, H: Exact and numerical solutions for non-linear Burger's equation by vim. Math. Comput. Model. 49, 1394-1400 (2009)

43. Inc, M: The approximate and exact solutions of the space- and time-fractional Burgers equations with initial conditions by variational iteration method. J. Math. Anal. Appl. 345, 476-484 (2008)

44. Repaci, A: Nonlinear dynamical systems: on the accuracy of Adomian's decomposition method. Appl. Math. Lett. 3(3), 35-39 (1990)

45. He, JH: A new approach to nonlinear partial differential equations. Int. J. Non-Linear Mech. 2(4), 203-205 (1997)

46. He, JH: A variational iteration approach to nonlinear problems and its applications. Mech. Appl. 20(1), 30-31 (1998)

47. $\mathrm{He}, \mathrm{JH}$ : Variational iteration method-a kind of nonlinear analytical technique: some examples. Int. J. Non-Linear Mech. 34, 708-799 (1999)

48. Liu, HM: Generalized variational principles for ion acoustic plasma waves by He's semi-inverse method. Chaos Solitons Fractals 23(2), 573-576 (2005)

49. Odibat, Z, Momani, S: The variational iteration method: an efficient scheme for handling fractional partial differential equations in fluid mechanics. Comput. Math. Appl. 58, 2199-2208 (2009)

50. Allahviranloo, T, Abbasbandy, S, Behzadi, SS: Solving nonlinear fuzzy differential equations by using fuzzy variational iteration method. Soft Comput. 18(11), 2191-2200 (2014)

51. Rouhparvar, $\mathrm{H}$, Abbasbandy, S: Numerical solution of the uncertain characteristic Cauchy reaction-diffusion equation by variational iteration method. Walailak J. Sci. Technol. 11(11), 987-998 (2014)

\section{Submit your manuscript to a SpringerOpen ${ }^{\circ}$ journal and benefit from:}

- Convenient online submission

- Rigorous peer review

- Open access: articles freely available online

- High visibility within the field

- Retaining the copyright to your article

Submit your next manuscript at $\gg$ springeropen.com 